\title{
Suppression of tissue inhibitor of metalloproteinase-1 by recombinant adeno-associated viruses carrying siRNAs in hepatic stellate cells
}

\author{
MIN CONG $^{1}$, TIANHUI LIU ${ }^{1}$, PING WANG $^{1}$, YONG XU $^{1}$, SHUZHEN TANG $^{1}$, \\ BAOEN WANG ${ }^{1}$, JIDONG JIA ${ }^{1}$, YONG LIU ${ }^{2}$, PAUL L. HERMONAT ${ }^{2}$ and HONG YOU ${ }^{1}$ \\ ${ }^{1}$ Liver Research Center, Beijing Friendship Hospital, Capital Medical University, \\ Beijing 100050, P.R. China; ${ }^{2}$ Gene Therapy Center, Department of Internal Medicine, \\ University of Arkansas for Medical Sciences, Little Rock, AR 72205, USA
}

Received July 15, 2009; Accepted August 24, 2009

DOI: 10.3892/ijmm_00000280

\begin{abstract}
Elevated tissue inhibitor of metalloproteinase (TIMP)-1 expression contributes to excess production of extracellular matrix in liver fibrosis. However, there are few studies on sustained suppression of TIMP-1. We aimed to construct a recombinant adeno-associated virus (AAV) carrying small interfering RNAs (siRNAs) of TIMP-1 and investigate the long-term effects of RNA interference upon the TIMP-1 gene in rat hepatic stellate cells (HSCs). Five siRNA oligomers targeting rat TIMP-1 were designed and transfected into HSCs. A U6 promoter followed by the siRNA which had the strongest suppression effect was cloned into the AAV vector and packed into 293 cells to construct the recombinant AAV/ siRNA-TIMP-1/neo. After infecting HSCs with this recombinant AAV, the transcription and expression levels of the TIMP-1 and matrix metalloproteinase-13 (MMP-13) genes were detected at 4 and 12 weeks. Three of the five designed siRNA oligomers had a suppressing effect on TIMP-1 expression in rat HSCs within $72 \mathrm{~h}$. The transcription and expression levels of TIMP-1 were suppressed significantly $(\mathrm{P}<0.05)$ following recombinant AAV/siRNA1-TIMP-1/neo infection and lasted 12 weeks. TIMP-1 expression in rAAV/ siRNA1-TIMP-1/neo-infected HSCs was suppressed by $60 \%$ after four weeks and $90 \%$ after twelve weeks when compared to the control recombinant AAV/neo and uninfected HSCs. Furthermore, the transcription and protein expression levels of MMP-13, the main substrate of TIMP-1, were elevated by $\sim 40 \%$ at twelve weeks in rAAV/siRNA-TIMP-1/neo-infected HSCs. RNA interference
\end{abstract}

Correspondence to: Dr Hong You, Liver Research Center, Beijing Friendship Hospital, Capital Medical University, Beijing 100050, P.R. China

E-mail: youhong30@hotmail.com

Key words: adeno-associated virus, small interfering RNA, tissue inhibitor of metalloproteinase-1 exerts suppressive effect on the TIMP-1 gene in cultured HSCs for a longer time when a recombinant AAV is utilized as the gene delivery vector.

\section{Introduction}

Liver fibrosis represents the final common pathological outcome for the majority of chronic liver insults. Hepatic stellate cells (HSCs) have now been clearly identified as the primary cellular source involved in the pathogenesis of liver fibrosis. During the development of liver fibrosis, stellate cells undergo activation, a process characterized by increased cell proliferation, morphological transformation into myofibroblast-like cells and synthesis of excessive extracellular matrix (ECM) components (1). These cells are also able to synthesize enzymes, called matrix metalloproteinases (MMPs), which can degrade matrix proteins in the extracellular space. Matrix degradation induced by MMPs is kept in check by concurrently secreted tissue inhibitors of metalloproteinases (TIMPs), powerful inhibitors of MMPs, especially TIMP-1. Studies (2-4) have shown that liver fibrosis can be attenuated by manipulating the TIMP-MMP balance. If expression of TIMP-1 in activated HSCs was inhibited, it should be possible to harness the latent matrix degrading capacity of a fibrotic or cirrhotic liver to facilitate matrix degradation, resulting in a return to normal or near normal architecture (5).

Our early study (6) had compared the suppressive effects of antisense RNA and small interfering RNA (siRNA) targeting the TIMP-1 gene in HSCs. Our results confirmed that, RNA interference can exert strong suppression of the target gene, whilst antisense RNA had no significant inhibitory effects on the TIMP-1 gene. These results were also observed in mice. The low efficiency of antisense oligonucleotides was most probably due to their lower resistance to nuclease degradation (7). Permanent expression of siRNA has been most frequently achieved by the use of virus-based delivery systems, therefore an adeno-associated virus (AAV) was chosen to carry the TIMP-1 short hairpin RNA (shRNA) because AAVs are safe, non-pathogenic, easier in application and extremely stable (8). The aim of this 
study was to construct a recombinant AAV carrying a siRNA of TIMP-1 and investigate the long-term effects of RNA interference on TIMP-1, and the elevation of MMP in rat HSCs.

\section{Materials and methods}

Cell culture. A rat HSC line (T6; donated by Dr Scott L. Friedman, USA) was cultured in Dulbecco's modified Eagle's medium (DMEM; Invitrogen, USA), supplemented with 10\% fetal bovine serum (FBS; Invitrogen), $10 \mathrm{U} / \mathrm{ml}$ penicillin (Sigma, USA) and $0.1 \mathrm{mg} / \mathrm{ml}$ streptomycin (Sigma). Cell cultures were grown at $37^{\circ} \mathrm{C}, 5 \% \mathrm{CO}_{2}$ in a humidified incubator and regularly passaged to maintain growth.

siRNA synthesis. Five siRNA duplexes were designed according to an online tool for siRNA design (Ambion company: www.ambion.com/techlib/misc/siRNA_finder. html). The selected sequence was screened by a BLAST search against all known rat genes to verify that only rat TIMP-1 mRNA was targeted. The siRNA duplexes were obtained from GeneChem Company (Shanghai, China) and information regarding these five siRNAs is summarized in Table I. Each RNA contained two additional deoxythymidine nucleotides at the $3^{\prime}$ end. A mismatched siRNA non-silencer was used as negative control and a fluorescence-labeled siRNA duplex, non-silencer-FITC, was also obtained to detect transfection efficiency.

siRNA transfection. A day before the experiment cells were seeded in 6-well plates at a density of $2 \times 10^{5}$ cells per well, aiming for $50 \%$ confluency on the day of the experiment. Transfection of the RNA oligonucleotides was performed using Lipofectamine 2000 (Invitrogen) following the manufacturer's instructions. A final RNA oligonucleotide concentration of $50 \mathrm{nM}$ was empirically determined to be the optimum transfection concentration. Transfected cells were incubated for $6 \mathrm{~h}$ at $37^{\circ} \mathrm{C}$, washed with PBS and then incubated in $2 \mathrm{ml}$ DMEM containing 10\% FBS. Twenty-four hours following transfection, the HSCs transfected with nonsilencer-FITC were harvested and passed through a fluorescence activated cell sorter (FACS) to determine transfection efficiency. HSCs transfected with the other 5 siRNAs and non-silencer were collected at 24,48 and $72 \mathrm{~h}$ post-transfection for additional analyses.

Western immunoblot analysis for TIMP-1. Cell pellets harvested at 24, 48 and $72 \mathrm{~h}$ were resuspended in cell lysis buffer [50 mM Tris (pH 7.4), $150 \mathrm{mM} \mathrm{NaCl}, 40 \mathrm{mM} \mathrm{NaF}$, $5 \mathrm{mM}$ EDTA, $5 \mathrm{mM}$ EGTA, $0.5 \%$ deoxycholic acid, $0.1 \%$ SDS] containing freshly added phenylmethylsulfonyl fluoride and a protease inhibitor mixture (Sigma). Cell lysates were incubated at $4^{\circ} \mathrm{C}$ for $20 \mathrm{~min}$ and centrifuged for $10 \mathrm{~min}$ at $4^{\circ} \mathrm{C}$. Protein concentration was determined using BCA protein assay kit (Pierce catalog \# 23227, USA). Equivalent amounts of protein $(10 \mu \mathrm{g})$ from each cell lysate were resolved using SDS-PAGE and 15\% acrylamide gels. Gels were electroblotted onto nitrocellulose membranes and incubated in blocking solution (TBS, Tween-20 0.1\% v/v, non-fat dry milk powder $5 \% \mathrm{w} / \mathrm{v}$ ) for $2 \mathrm{~h}$ at room temperature. Following blocking the membrane was incubated with a mouse antiTIMP-1 monoclonal antibody diluted 1:1000 (Catalogue number MAB580; R\&D Systems, USA) overnight at $4^{\circ} \mathrm{C}$. The membrane was washed in TBS supplemented with Tween-20 $(\mathrm{TBS}-\mathrm{T} ; 0.1 \% \mathrm{v} / \mathrm{v})$ three times then incubated with a horseradish peroxidase-conjugated goat anti-mouse antibody (Sigma) diluted 1:2000 for $1 \mathrm{~h}$ at room temperature. Membranes were washed three times with TBS-T and protein bands detected using enhanced chemiluminescence technology (SuperSignal West Pico Chemiluminescent Substrate; Pierce). The signal intensity of visible protein bands was quantified by densitometry on a Bio-Rad Gel Doc 2000 gel documentation system with the Quantity One ${ }^{\circledR}$ software package. The positive control used in the method was ß-actin.

Plasmid construction. After transfection with siRNAs, we screened one pair of siRNAs which could effectively inhibit expression of the TIMP-1 gene in HSC-T6 and reconstructed an AAV vector containing this specific sequence to form shRNA. The mouse U6 promoter, a promoter of RNA polymerase III, was cloned by the following primers using mouse genomic DNA as template: forward primer, 5'-AGAT CTCGACGCCGCCATCTCTAGG-3' (underlined sequence denotes a $B g l I I$ site); reverse primer, 5'-AAACAAGGCTTT TCTCCAAGGG-3'. The PCR consisted of 30 cycles at $94^{\circ} \mathrm{C}$ for $45 \mathrm{sec}, 56^{\circ} \mathrm{C}$ for $40 \mathrm{sec}$ and $72^{\circ} \mathrm{C}$ for $30 \mathrm{sec}$. After amplification, the U6 promoter was cloned into a pGMT vector, a mouse U6 promoter-driven shRNA expression cassette was obtained by PCR using the recombinant pGMT vector as the template. We used the same forward primer that was employed in amplifying the U6 promoter. The reverse primer contained the same sequence used above but also had a $21 \mathrm{nt}$ sense-specific sequence targeting TIMP-1, reverse complementary sequences separated by a $9 \mathrm{nt}$ loop and terminated by a six-thymidine termination signal. The resulting reverse primer sequence was 5'-CTCGAGAAAAAAGGGC TAAATTCATGGGTTCCCTCTCTTGAAGGGAACCCAT GAATTTAGCCCAAACAAGGCTTTTCTCCAAGGG-3' (underlined sequence denotes a $\mathrm{XhoI}$ site). The PCR consisted of 30 cycles at $94^{\circ} \mathrm{C}$ for $45 \mathrm{sec}, 60^{\circ} \mathrm{C}$ for $40 \mathrm{sec}$ and $72^{\circ} \mathrm{C}$ for $45 \mathrm{sec}$. The PCR product was inserted into the $B g l \mathrm{II}$ and XhoI sites of a pGMT-vector. The final clone selected was verified by sequencing. The mouse U6 promoter, hairpin sequence and terminator sequences were cut out using $B g l \mathrm{II}$ and XhoI restriction enzymes and ligated into an AAV vector-pdl6-95/ neo (donated by Dr Paul L. Hermonat) to construct the recombinant AAV (rAAV) pdl6-95/siRNATIMP-1/neo.

Viral production and purification. The rAAV/siRNA-TIMP-1/ neo and rAAV/neo virus stocks were generated from 293 cells by transfecting 293 cells with $5 \mu \mathrm{g}$ of the pdl6-95/siRNATIMP-1/neo or pdl6-95/neo vector along with $5 \mu \mathrm{g}$ of the AAV/Ad complementor plasmid pSH3. After DNase I treatment $(100 \mathrm{U} / \mathrm{ml}$ for $1 \mathrm{~h})$, the virus solution was applied to a heparin-agarose column (Sigma). The matrix was washed, eluted with phosphate-buffered saline and concentrated. Each virus stock was titered by dot blot and determined to be at $\sim 1 \times 10^{8}$ encapsidated genomes per milliliter. 
Table I. siRNA duplex sequences used to target at rat TIMP-1.

\begin{tabular}{lll}
\hline Code & \multicolumn{1}{c}{ Sense (5'-3') } & Antisense (5'-3') \\
\hline $1(161-181)$ & GGGCUAAAUUCAUGGGUUCCCdtdt & GGGAACCCAUGAAUUUAGCCCdtdt \\
$2(190-208)$ & UCAUCGAGACCACCUUAUAdtdt & UAUAAGGUGGUCUCGAUGAdtdt \\
$3(208-226)$ & ACCAGCGUUAUGAGAUCAAdtdt & UUGAUCUCAUAACGCUGGUdtdt \\
$4(226-244)$ & AGAUGACUAAGAUGCUCAAdtdt & UUGAGCAUCUUAGUCAUCUdtdt \\
$5(445-463)$ & AGGCCUUCGUAAAGACCUAdtdt & UAGGUCUUUACGAAGGCCUdtdt \\
$6($ Control $)$ & UUCUCCGAACGUGUCACGUdtdt-FITC & ACGUGACACGUUCGGAGAAdtdt \\
\hline
\end{tabular}

Virus infection. Twenty-four hours prior to infection, HSC-T6 were trypsinized and plated at a density of $2 \times 10^{5}$ cells per well in 6-well plates, in DMEM containing $10 \%$ FBS. Before infection, the cells were washed twice with DMEM, then incubated in a mixture of $1 \mathrm{ml} \mathrm{rAAV/siRNA-TIMP-1/neo}$ and $1 \mathrm{ml}$ DMEM without FBS, or $1 \mathrm{ml} \mathrm{rAAV} /$ neo and $1 \mathrm{ml}$ DMEM without FBS as a mock control. Cells were incubated with these mixtures for $6 \mathrm{~h}$ at $37^{\circ} \mathrm{C}$. After infection, cells were washed with PBS and incubated in $2 \mathrm{ml}$ DMEM containing $10 \%$ FBS. Two days later, cells were washed and resuspended in DMEM with $10 \%$ FBS containing $500 \mu \mathrm{g} / \mathrm{ml}$ neomycin and maintained for 4 weeks and 12 weeks.

Detection of transcription and expression levels of TIMP-1 and MMP-13 in infected HSC-T6. Because rat MMP-13 is tightly regulated by TIMP-1, the transcription and expression levels of both TIMP-1 and MMP13 were monitored after rAAV infection. Total cellular RNA was isolated by TRIzol (Invitrogen) extraction according to the manufacturer's protocol. The reverse transcription reaction was carried out using a reverse transcription kit (Promega, USA) and $1 \mu \mathrm{g}$ of RNA treated with DNase I (Promega). Real-time quantitative PCR for the detection of TIMP-1 was done using a method established by our laboratory (9). RNA normalization was performed against GAPDH and primer sequences for the TIMP-1 real-time quantitative PCR were: TIMP-1 forward primer, 5'-CCTGCCAAGTATGATGACATCAAGA-3'; TIMP-1 reverse primer, 5'-GTAGCCCAGGATGCCCTTT AGT-3'; TIMP-1 probe, 5'-FAM-TGGTGAAGCAGGC GGCCGAG-TAMRA-3'. Primers used for amplifying MMP13 and GAPDH were as follows: MMP-13 forward primer, 5'-GAACCACGTGTGGAGTTATG-3'; MMP-13 reverse primer, 5'-AGCCACGCATAGTCATGTAG-3'; GAPDH forward primer, 5'-GACATCAAGAAGGTGGTG AAGC-3'; GAPDH reverse primer, 5'-CTTGCTCTCAGTA TCCTTGC-3'. PCR consisted of 30 cycles at $94^{\circ} \mathrm{C}$ for $45 \mathrm{sec}$, $54^{\circ} \mathrm{C}$ for $40 \mathrm{sec}$ and $72^{\circ} \mathrm{C}$ for $30 \mathrm{sec}$.

Following infection, cell lysates were used for the detection of TIMP-1, MMP-13 and ß-actin by Western blot analysis as described.

\section{Results}

Identification of effective siRNAs for inhibition of TIMP-1 and construction of a rAAV/siRNA-TIMP-1/neo vector. We constructed an AAV vector containing a specific sequence which could effectively inhibit expression of the TIMP-1
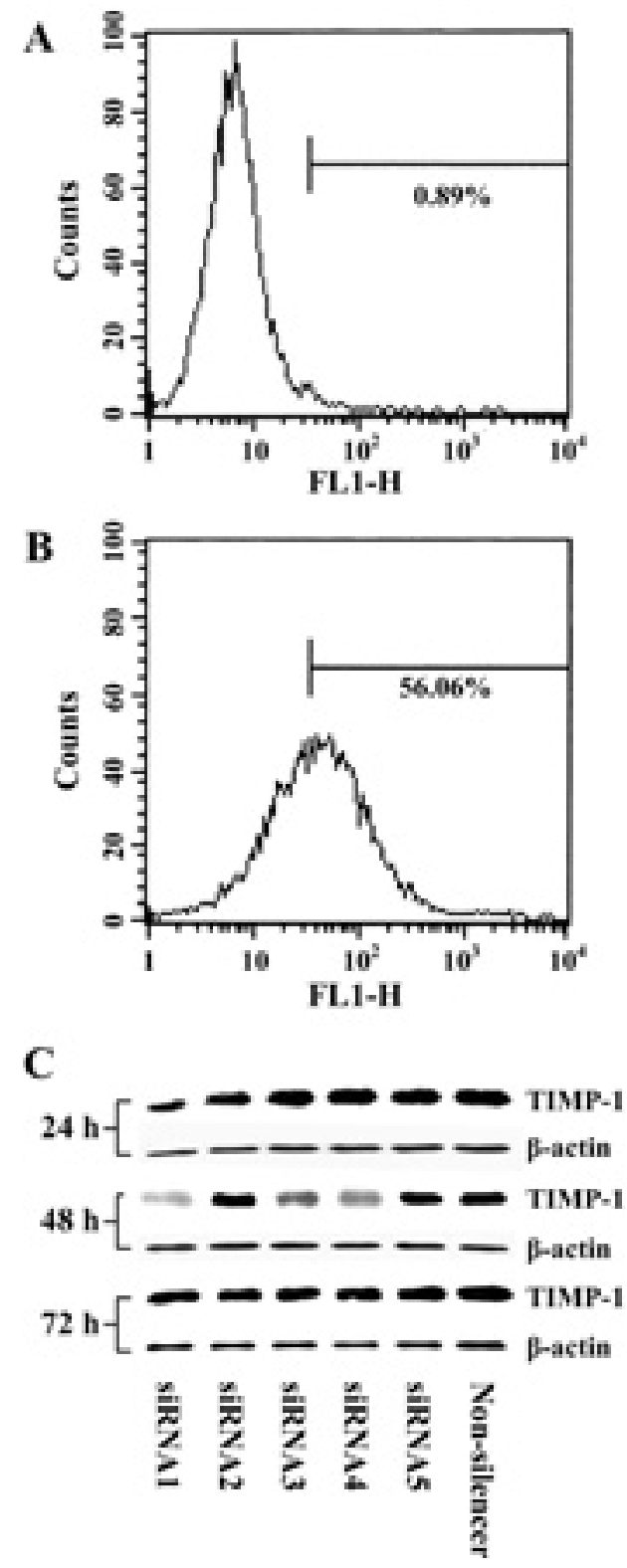

Figure 1. Silencing of TIMP-1 gene expression by synthetic siRNAs. FACS results of control (A) and transfection efficiency of $50 \mathrm{nmol} / 1$ non-silencerFITC (B) are shown. The transfection efficiency of $50 \mathrm{nmol} / 1$ non-silencerFITC reached $56.06 \%$. (C) Protein expression of TIMP-1 at 24, 48 and $72 \mathrm{~h}$ post-transfection of siRNAs. B-actin content was detected as a loading control. The Fig. shows representative Western blotting for TIMP-1 and $\beta$-actin. The signals were detected at $28 \mathrm{kDa}$ for TIMP-1 and $45 \mathrm{kDa}$ for B-actin. Three of five siRNA duplexes, siRNA1, siRNA3 and siRNA4 suppressed TIMP-1 expression at $48 \mathrm{~h}$ post-transfection and siRNA1 had the strongest suppressive effect. At $72 \mathrm{~h}$ post-transfection, all five siRNAs had no significant suppressive effect compared with the non-silencer. 

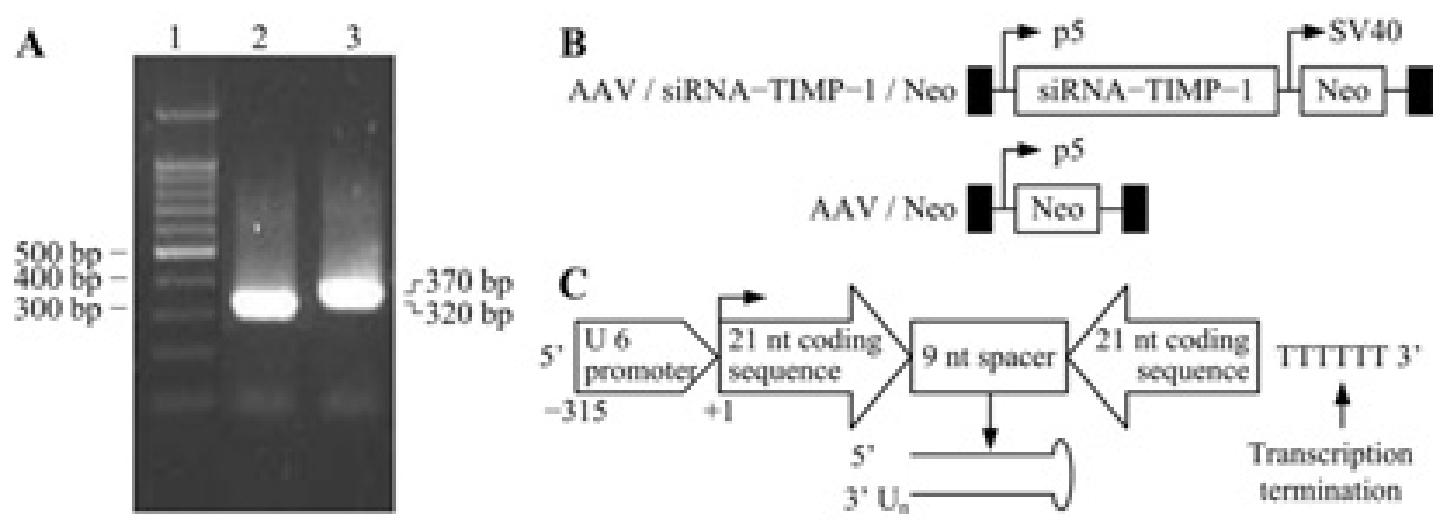

Figure 2. Strategy for reconstruction of rAAV/siRNA-TIMP-1/neo. (A) Lane 1 represents 100 bp marker; lane 2 represents PCR product of amplifying mouse U6 promoter $(320 \mathrm{bp}$ ); lane 3 represents PCR product of amplifying U6-siRNA-TIMP-1, in which shRNA expression cassette was followed by U6 promoter (370 bp). (B) Shown are the structures of the rAAV/siRNA-TIMP-1/neo and rAAV/neo vectors. The names are indicated to the left of the vector structural map. Also shown are the terminal repeats (TR) as black rectangles. (C) Schematic representation of a shRNA expression cassette. The U6-RNA promoter was cloned in front of the TIMP-1 gene-specific targeting sequence (21 nt sequences from the target transcript separated by a 9 nt loop from the reverse complement of the same sequence) and six-thymidine residues (T6) as a termination signal. The secondary structure of the transcript is shown and it is believed to be further cleaved by Dicer to generate a $21 \mathrm{nt}$ siRNA that forms a dsRNA-endonuclease complex and will bind and destroy TIMP-1 mRNA.

gene. Five independent siRNA molecules were designed and synthesized. The transfection efficiencies of 20,50 and $100 \mathrm{nM}$ non-silencer-FITC were observed and $50 \mathrm{nM}$ was determined to be the optimum transfection concentration (data not shown). FACS results of the HSC-T6 transfected with non-silencerFITC displayed transfection efficiencies that reached $50 \%$ and beyond (Fig. 1A and B). Western blot analysis of whole-cell extracts from HSC-T6 prepared at 24, 48 and $72 \mathrm{~h}$ posttransfection with a $50 \mathrm{nM}$ concentration of the five siRNA duplexes and non-silencer as control was performed. Equal protein amounts were loaded and probed with antibodies specific for rat TIMP-1 and B-actin. Expression of TIMP-1 was not affected by the 5 synthetic siRNAs at $24 \mathrm{~h}$ posttransfection. However, at $48 \mathrm{~h}$ post-transfection we observed a reduction in TIMP-1 expression levels of up to $50 \%$ in three of the five siRNA duplexes. The three effective duplexes were targeting rat TIMP-1 at nt 161-181 (siRNA1), nt 208-226 (siRNA3) and nt 226-244 (siRNA4). The expression of TIMP-1 was dramatically reduced by $\sim 80 \%$ at $48 \mathrm{~h}$ posttransfection by siRNA1. The remaining two siRNAs, targeting rat TIMP-1 at nt 190-208 (siRNA2) and nt 445-463 (siRNA5) had no significant effect on TIMP-1 expression. At $72 \mathrm{~h}$ post-transfection, expression of TIMP-1 in the HSC-T6 did not appear to be significantly affected by the 5 siRNA duplexes or the non-silencer (Fig. 1C), indicating that this suppressive effect by synthetic siRNAs is short term. Due to its strong inhibitory effect, siRNA1 was chosen for further study.

An AAV gene delivery vector was then constructed by ligating the siRNA1 sequence downstream of the mouse U6 promoter. The mouse U6 promoter was generated by PCR using the primers described in the Materials and methods. The U6 promoter followed by a $21 \mathrm{nt}$ sense-specific sequence targeting TIMP-1 and reverse complementary sequences separated by a $9 \mathrm{nt}$ loop and terminated by a six-thymidine termination signal was amplified by PCR (Fig. 2A). The structure of rAAV/siRNA-TIMP-1/neo is shown in Fig. 2B. This vector employs the U6 promoter to express shRNAs in the form of an inverted repeat sequence containing a hairpin loop allowing the shRNA to be processed into siRNA within the cell (Fig. 2C). PCR, restriction enzyme digestion and DNA sequencing confirmed the structure of dl6-95/siRNA1TIMP-1/ neo. Subsequently, rAAV/siRNA1-TIMP-1/neo and $\mathrm{rAAV} /$ neo virus stocks were generated from 293 cells and were titered by dot blot and determined to be $\sim 1 \times 10^{8}$ encapsidated genomes per milliliter (data not shown).

Suppression of TIMP-1 expression in HSC-T6 by rAAV/ siRNA-TIMP-1/neo transduction. The mRNA expression levels of TIMP-1 in transduced and non-transduced HSC-T6 was quantified by real-time PCR at 4 and 12 weeks. The input RNA amount was normalized by GAPDH PCR. Fig. 3 shows the expression of TIMP-1 mRNA transcripts in infected and G418-selected cells at various time points after rAAV/ siRNA1-TIMP-1/neo and rAAV/neo infection. The results demonstrate mRNA expression levels of TIMP-1 in rAAV/ siRNA1-TIMP-1/neo-infected HSC-T6 was dramatically suppressed compared to the cells infected with $\mathrm{rAAV} /$ neo and control uninfected cells even after 12 weeks $(\mathrm{P}<0.05)$. At 4 and 12 weeks, the mRNA expression levels of TIMP-1 in rAAV/siRNA1-TIMP-1/neo-infected HSC-T6 were suppressed but had not significantly changed between these time points. Down-regulation of TIMP-1 expression by AAV delivered siRNA was analyzed by Western blot and is shown in Fig. 4. Cell lysates were prepared 4 and 12 weeks after infection and used for the detection of TIMP-1 and B-actin. The results demonstrate protein expression levels of TIMP-1, at both 4 and 12 weeks, in rAAV/siRNA-TIMP-1/neo-infected HSC-T6 had notably decreased when compared to cells infected with $\mathrm{rAAV} /$ neo or the control $(\mathrm{P}<0.05)$. Cells infected with $\mathrm{rAAV} / \mathrm{siRNA}$-TIMP-1/neo exhibited an $\sim 60 \%$ reduction in TIMP-1 expression after 4 weeks, increasing to a $>90 \%$ reduction at 12 weeks post-transfection.

Increased MMP-13 expression in HSC-T6 infected with $r A A V /$ siRNA-TIMP-1/neo. We quantified mRNA and protein expression levels of MMP-13 in rAAV/siRNA-TIMP1/ neo-infected HSCs by RT-PCR and Western blot analysis at 

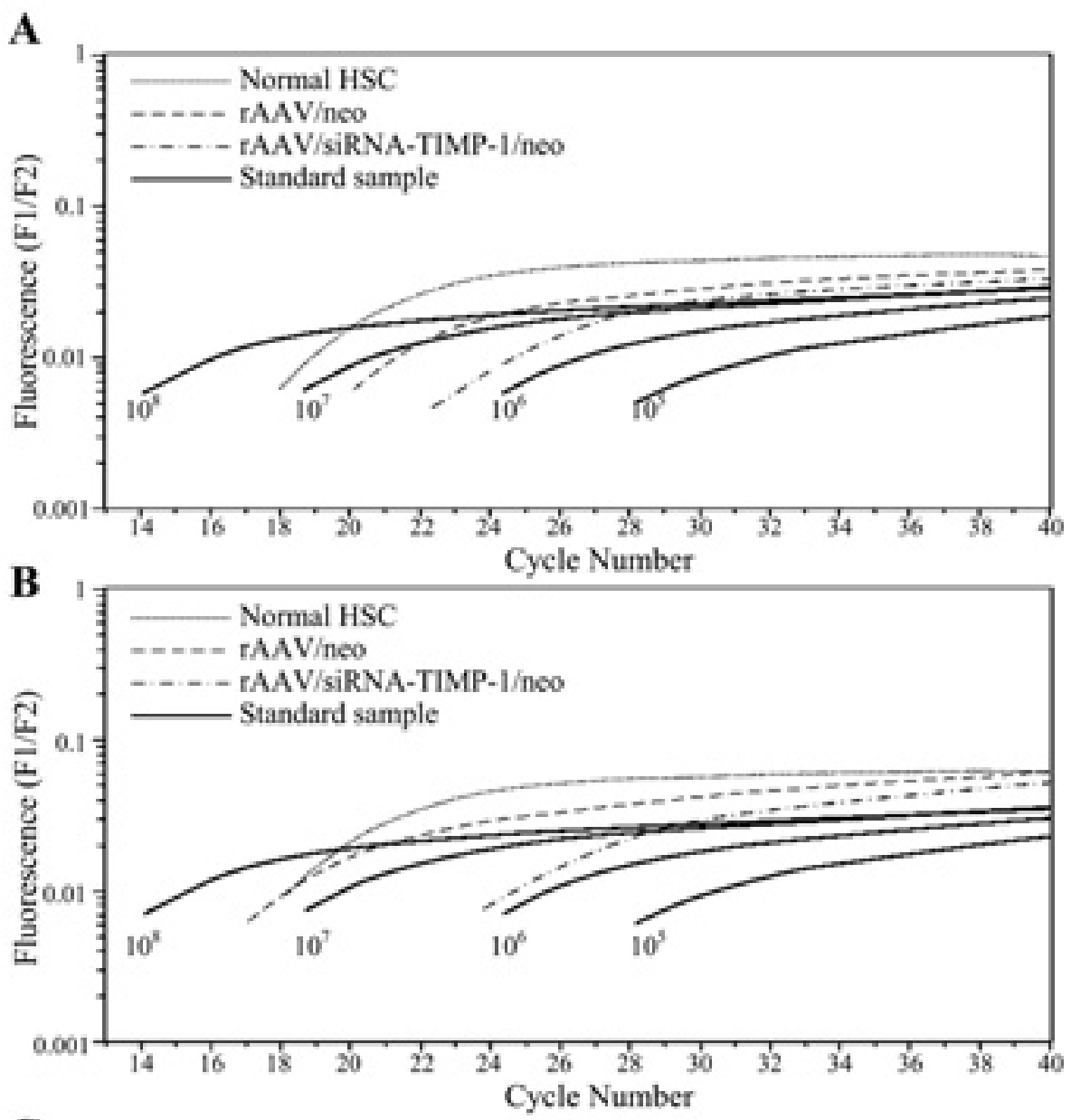

C

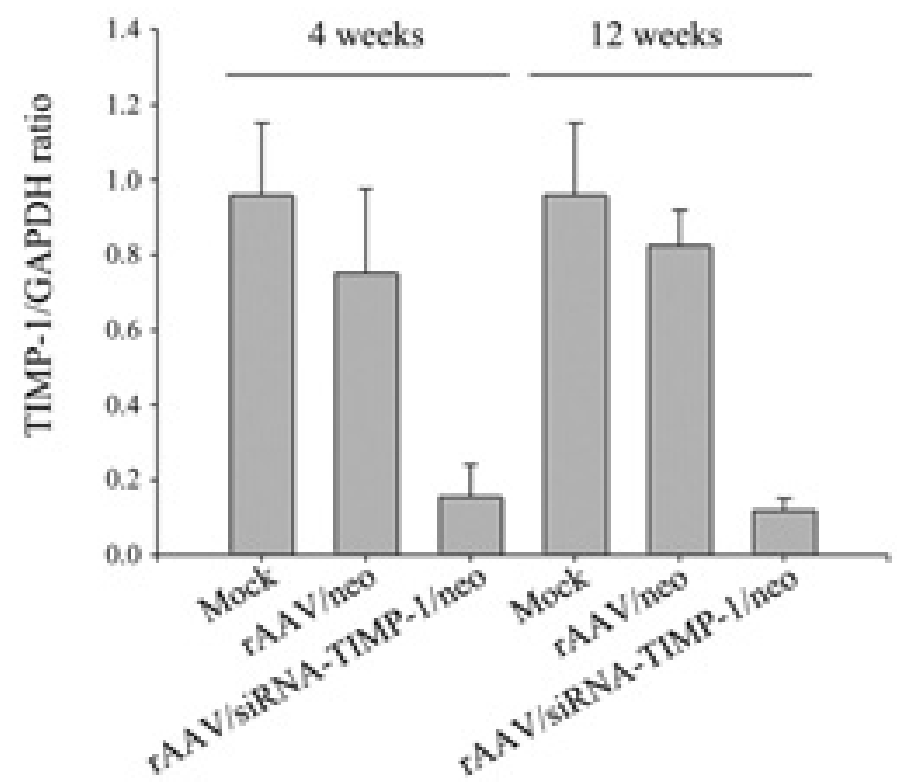

Figure 3. Suppression of TIMP-1 RNA expression in HSC-T6 infected with rAAV. (A and B) Representative results for mRNA expression levels of TIMP-1 in infected HSC-T6 and uninfected cells quantified by real-time PCR after reverse transcription of total RNA at 4 and 12 weeks post-infection. Standard curves were made by using a standard sample containing $10^{5}-10^{8}$ copies of a TIMP-1 plasmid as template. (C) Results are expressed as relative to the transcription level of GAPDH in rAAV-infected HSCs and uninfected cells and presented as the mean \pm standard error derived from three independent experiments. mRNA expression levels of TIMP-1 in HSC-T6 infected with rAAV/siRNA1-TIMP-1/neo was dramatically suppressed compared with to rAAV/neo-infected cells at 4 and 12 weeks post-infection $(\mathrm{P}<0.05)$.

12 weeks post-infection. Input RNA and protein was normalized to GAPDH and $\beta$-actin, respectively. The mRNA expression level of MMP-13 in rAAV/siRNA1-TIMP-1/neo- infected HSC-T6 was greatly increased when compared to cells infected with $\mathrm{rAAV} / \mathrm{Neo}$ or uninfected cells $(\mathrm{P}<0.05$; Fig. 5A). Protein expression of MMP-13 in rAAV/siRNA1- 
$\mathbf{A}$

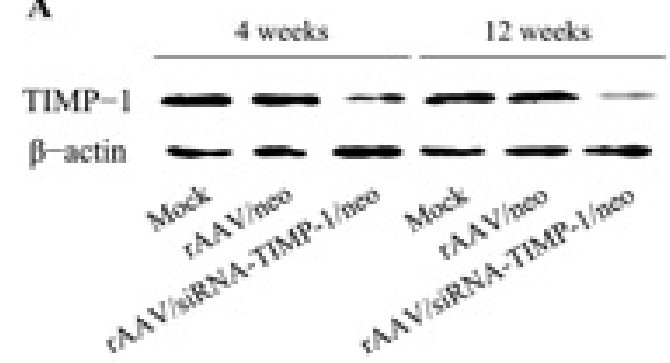

B

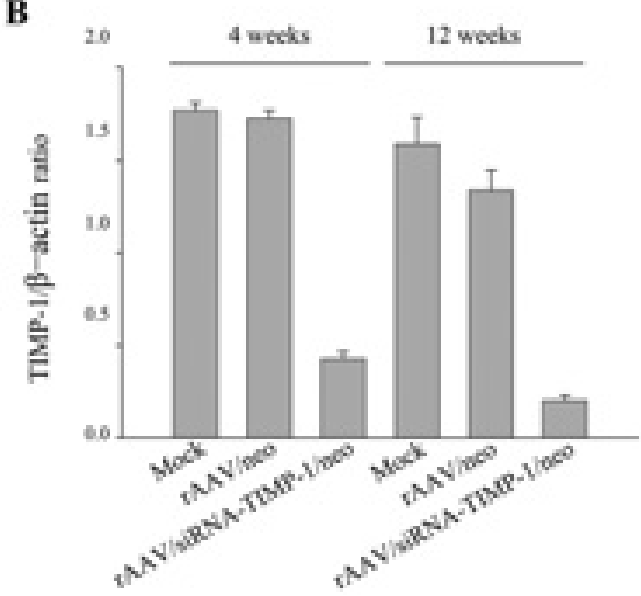

Figure 4. Suppression of TIMP-1 protein expression in HSC-T6 by rAAV (A) Representative Western blotting for TIMP-1 and $B$-actin in rAAV-infected cells and uninfected cells. B-actin content was detected as a loading control. The signals were detected at $28 \mathrm{kDa}$ for TIMP- 1 and $45 \mathrm{kDa}$ for $\beta$-actin (B) Results are expressed as relative to the expression level of $B$-actin in rAAV-infected and uninfected HSCs and presented as the mean \pm standard error derived from three independent experiments. The TIMP-1 expression level in HSCs infected with rAAV/siRNA-TIMP-1/neo at 12 weeks postinfection was reduced by $>90 \%$ compared $\mathrm{rAAV} /$ neo-infected HSCs, while the same recombinant AAV inhibited $60 \%$ of TIMP-1 protein expression at 4 weeks post-infection.

TIMP-1/neo-infected cells was elevated by $\sim 40 \%$ at 12 weeks post-infection (Fig. 5B), additionally, there was no significant morphological change between the different groups of infected cultures (Fig. 5C).

\section{Discussion}

In fibrotic liver there is net deposition of fibrillar matrix that is comprised predominantly of the interstitial collagens, types I and III. These triple helical molecules are cleaved at a specific Gly-Ile/Leu site in their $\alpha$-chain by interstitial collagenases (MMP-1 in humans, MMP-13 in rats). The active forms of these MMPs can be inhibited by all of the TIMPs, which are important regulatory molecules in tissue remodeling and repair. Both TIMP-1 and TIMP-2 have been identified in the liver, but the role of TIMP-1 is more important than that of TIMP-2 in hepatic pathological process because MMP1/ MMP13 is tightly regulated by TIMP-1 (10). In the present study, siRNA technology was utilized for attaining a stronger inhibition of TIMP-1 function in HSC-T6 in vitro, hopefully developing a foundation for its application in vivo.

To find a siRNA sequence with the strongest suppressive effect for reconstructing $\mathrm{rAAV} / \mathrm{siRNA}$-TIMP-1/neo, five siRNA oligomers targeting rat TIMP-1 were synthesized and transfected into rat HSC-T6 initially. Although three of the five siRNAs exhibited the ability to inhibit at $48 \mathrm{~h}$ posttransfection, this effect was lost by $72 \mathrm{~h}$ post-transfection. These results demonstrated that oligonucleotide-mediated siRNA suffers from the limitation of short-term suppression of gene expression. By transfecting five siRNAs targeting rat TIMP-1 at different gene sites and comparing their effects, siRNA1 was chosen for further study because of its strong inhibitory activity.

In the present study, a U6 promoter-driven TIMP-1 shRNA cassette which included the siRNA1 sequence was

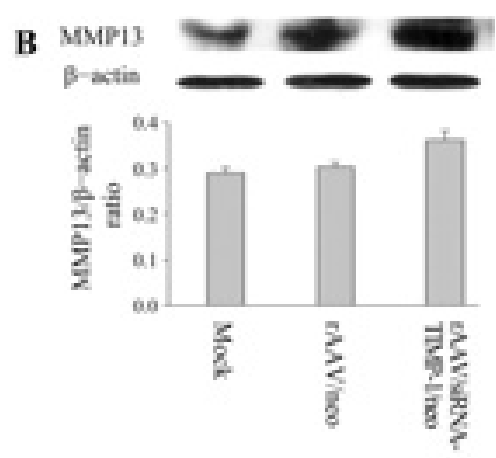

C

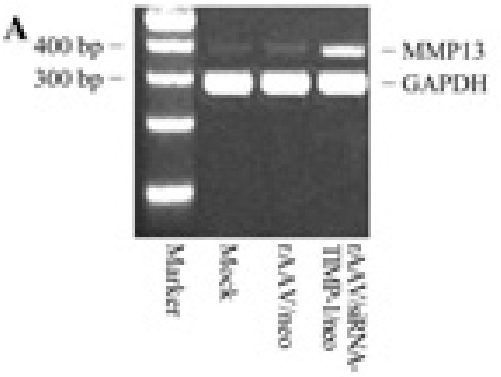

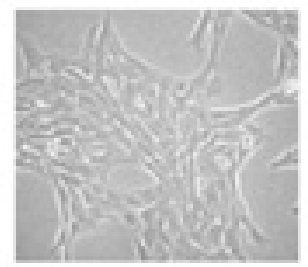

Mock

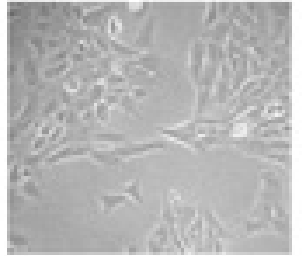

rAAV/neo

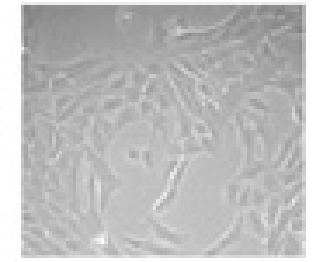

TAAV/siRNA-TIMP-1/neo

Figure 5. Increase of MMP-13 expression in HSC-T6 infected with rAAV. (A) mRNA expression levels of MMP-13 in infected and uninfected HSCs quantified by semi-quantitative PCR after reverse transcription of total RNA at 12 weeks post-infection showed mRNA levels of MMP-13 in rAAV/siRNA-TIMP-1/neoinfected HSC-T6 were increased. (B) Representative Western blotting for MMP-13 and $B$-actin in rAAV-infected cells and uninfected cells. The signals were detected at $46 \mathrm{kDa}$ for MMP-13 and $45 \mathrm{kDa}$ for B-actin. MMP13 expression levels in rAAV/siRNA-TIMP-1/neo-infected HSC-T6 were increased compared to rAAV/neo-infected cells $(\mathrm{P}<0.05)$. (C) Morphologic exhibition of normal HSCs, rAAV/neo- and rAAV/siRNA-TIMP-1/neo-infected cells. There was no significant change after infection with $\mathrm{rAAV} /$ neo or rAAV/siRNA-TIMP-1/neo after 12 weeks. 
reconstructed and packaged into $\mathrm{rAAV} / \mathrm{siRNA}$-TIMP-1/neo to acquire long-term inhibitory function. In addition, a virus control (rAAV/neo) was used to exclude possibilities that may complicate interpretation of the experimental data due to the employment of viral vector. The real-time PCR and Western blot analysis also demonstrated the lack of a global alteration in mRNA and protein levels as two housekeeping genes were unaffected by infection with shRNA-expressing vectors. Morphological analysis demonstrated that there was no detrimental effect on the morphology of infected cells. Our study shows that rAAV/siRNA-TIMP-1/neo can specifically inhibit the expression of TIMP-1 without deleterious function on the infected cells. In addition, we also detected the mRNA and protein expression of rat MMP-13 in HSCs at 12 weeks post-infection to investigate if MMP-13 expression was affected by TIMP-1. Our results demonstrated that both mRNA and protein expression of MMP-13 were elevated compared with a mock control $(\mathrm{P}<0.05)$, confirming that the suppression of TIMP-1 would elevate the expression of MMP-13, thereby facilitating matrix degradation. Further studies utilizing $\mathrm{rAAV} / \mathrm{siRNA}$-TIMP-1/neo in a rat liver fibrosis model are required to validate the anti-fibrotic effect in vivo.

The first and still the most extensive clinical experience concerning the administration of AAV vectors comes from the treatment of cystic fibrosis $(11,12)$. These investigations provided much of the safety profile of the vector and experience in human applications, paving the way for subsequent administration to other disorders such as hemophilia $(13,14)$, retinal diseases $(15,16)$, muscular dystrophy $(17,18)$, neurological diseases $(19,20)$ or cancer $(21,22)$. Our results suggest that AAV vectors also have the potential for the therapeutic delivery of siRNA. Manno et al (23) demonstrated the safety of intramuscular injections of AAVfactor IX in hemophilia patients due to the fact that none of the patients developed antibodies to factor IX and none showed evidence of vector sequence in the semen. Followup studies have continued for up to 6 years and there is still no evidence of tumor formation (24). These clinical trials present the possibility that AAV-mediated RNAi could be used in gene therapy. The highly specific and sustained reduction of TIMP-1 proteins and subsequently increased MMP-13 protein in HSCs due to rAAV/siRNA-TIMP-1/ neo infection may prove useful for treating liver fibrosis in vivo.

\section{Acknowledgements}

This study was supported by the Program Foundation for Excellent Scholars in Beijing (20071D0300100060), Major State Basic Research Development Program of China (2007CB512802) and National High Technology Research and Development Program of China (2006AA02A410). Municipal Key Laboratory of Beijing for Regulation of Liver Protection and Regeneration.

\section{References}

1. Friedman SL: The cellular basis of hepatic fibrosis: mechanisms and treatment strategies. N Engl J Med 328: 1828-1835, 1993.
2. Iimuro Y, Nishio T, Morimoto T, Nitta T, Stefanovic B, Choi SK, Brenner DA and Yamaoka Y: Delivery of matrix metalloproteinase-1 attenuates established liver fibrosis in the rat. Gastroenterology 124: 445-458, 2003.

3. Siller-López F, Sandoval A, Salgado S, Salazar A, Bueno M, Garcia J, Vera J, Gálvez J, Hernández I, Ramos M, AguilarCordova E and Armendariz-Borunda J: Treatment with human metalloproteinase- 8 gene delivery ameliorates experimental rat liver cirrhosis. Gastroenterology 126: 1122-1133, 2004.

4. Jiang W, Wang JY, Yang CQ, Liu WB, Wang YQ and He BM: Effects of a plasmid expressing antisense tissue inhibitor of metalloproteinase-1 on liver fibrosis in rats. Chin Med J (Engl) 118: 192-197, 2005.

5. Iredale JP: Cirrhosis: new research provides a basis for rational and targeted treatments. BMJ 327: 143-147, 2003.

6. Cong M, Wang P, Liu TH, Xu Y, Lu Y, Tang SZ, Liu XM, Wang BE, Jia JD and You H: Comparison between the suppression of tissue inhibitor of metalloproteinase-1 gene expression by recombinant adeno-associated virus carrying antisense RNA and small interfering RNA (siRNA) of TIMP-1 in rat hepatic stellate cells. Zhonghua Gan Zang Bing Za Zhi 14: 742-747, 2006.

7. Bertand JR, Pottier M, Vekris A, Opolon P, Maksimenko A and Malvy C: Comparison of antisense oligonucleotides and siRNAs in cell culture and in vivo. Biochem Biophys Res Commun 296: 1000-1004, 2002.

8. Mochizuki S, Mizukami H, Ogura1 T, Kure S, Ichinohe A, Kojima K, Matsubara Y, Kobayahi E, Okada T, Hoshika A, Ozawa $\mathrm{K}$ and Kume A: Long-term correction of hyperphenylalaninemia by AAV-mediated gene transfer leads to behavioral recovery in phenylketonuria mice. Gene Ther 11: 1081-1086, 2004.

9. Cong M, Yan ZY, Wang P, Zhang Y, Xu Y, Lu Y, Wang BE, Jia JD and You H: A sensitive, specific method of real-time quantitative PCR for detection of tissue inhibitor of metalloproteinase-1 gene. Chin J Lab Med 28: 533-537, 2005.

10. Okazaki I, Watanabe T, Hozawa S, Niioka M, Arai M and Maruyama K: Reversibility of hepatic fibrosis: from the first report of collagenase in the liver to the possibility of gene therapy for recovery. Keio J Med 50: 58-65, 2001.

11. Flotte TR: Recent developments in recombinant AAV mediated gene therapy for lung diseases. Curr Gene Ther 5: 361-366, 2005.

12. Leung K, Louca E, Munson K, Dutzar B, Anklesaria P and Coates AL: Calculating expected lung deposition of aerosolized administration of AAV vector in human clinical studies. J Gene Med 9: 10-21, 2007.

13. Davidoff AM, Gray JT, Ng CY, Zhang Y, Zhou J, Spence Y, Bakar Y and Nathwani AC: Comparison of the ability of adenoassociated viral vectors pseudotyped with serotype 2, 5, and 8 capsid proteins to mediate efficient transduction of the liver in murine and nonhuman primate models. Mol Ther 11: 875-888, 2005.

14. Manno CS, Pierce GF, Arruda VR, Glader B, Ragni M, Rasko JJ, Ozelo MC, Hoots K, Blatt P, Konkle B, Dake M, Kaye R, Razavi M, Zajko A, Zehnder J, Rustagi PK, Nakai H, Chew A, Leonard D, Wright JF, Lessard RR, Sommer JM, Tigges M, Sabatino D, Luk A, Jiang H, Mingozzi F, Couto L, Ertl HC, High KA and Kay MA: Successful transduction of liver in hemophilia by AAV-factor IX and limitations imposed by the host immune response. Nat Med 12: 342-347, 2006.

15. Min SH, Molday LL, Seeliger MW, Dinculescu A, Timmers AM, Janssen A, Tonagel F, Tanimoto N, Weber BH, Molday RS and Hauswirth WW: Prolonged recovery of retinal structure/function after gene therapy in an Rs1h-deficient mouse model of $x$-linked juvenile retinoschisis. Mol Ther 12: 644-651, 2005.

16. Dinculescu A, Glushakova L, Min SH and Hauswirth WW: Adeno-associated virus-vectored gene therapy for retinal disease. Hum Gene Ther 16: 649-663, 2005.

17. Qiao C, Li J, Zhu T, Draviam R, Watkins S, Ye X, Chen C, Li J and Xiao X: Amelioration of laminin-alpha2-deficient congenital muscular dystrophy by somatic gene transfer of miniagrin. Proc Natl Acad Sci USA 102: 11999-12004, 2005.

18. Lai Y, Yue Y, Liu M, Ghosh A, Engelhardt JF, Chamberlain JS and Duan D: Efficient in vivo gene expression by trans splicing adeno-associated viral vectors. Nat Biotechnol 23: 1435-1439, 2005.

19. Rodriguez-Lebron E, Denovan-Wright EM, Nash K, Lewin AS and Mandel RJ: Intrastriatal rAAV-mediated delivery of antihuntingtin shRNAs induces partial reversal of disease progression in R6/1 Huntington's disease transgenic mice. Mol Ther 12: 618-633, 2005. 
20. Eslamboli A, Georgievska B, Ridley RM, Baker HF, Muzyczka N, Burger C, Mandel RJ, Annett L and Kirik D: Continuous lowlevel glial cell line-derived neurotrophic factor delivery using recombinant adeno-associated viral vectors provides neuroprotection and induces behavioral recovery in a primate model of Parkinson's disease. J Neurosci 25: 769-777, 2005.

21. Liu G, Martins IH, Chiorini JA and Davidson BL: Adenoassociated virus type 4 (AAV4) targets ependyma and astrocytes in the subventricular zone and RMS. Gene Ther 12: 1503-1508, 2005.

22. Isayeva $\mathrm{T}, \mathrm{Ren} \mathrm{C}$ and Ponnazhagan S: Recombinant adenoassociated virus 2-mediated antiangiogenic prevention in a mouse model of intraperitoneal ovarian cancer. Clin Cancer Res 11: 1342-1347, 2005.
23. Manno CS, Chew AJ, Hutchison S, Larson PJ, Herzog RW, Arruda VR, Tai SJ, Ragni MV, Thompson A, Ozelo M, Couto LB, Leonard DG, Johnson FA, McClelland A, Scallan C, Skarsgard E, Flake AW, Kay MA, High KA and Glader B: AAV-mediated factor IX gene transfer to skeletal muscle in patients with severe hemophilia B. Blood 101: 2963-2972, 2003.

24. High KA: Clinical gene transfer studies for hemophilia B. Semin Thromb Hemost 30: 257-267, 2004. 